\title{
HRCT Sings Analysis of Pulmonary Mucinous Carcinoma
}

\section{Haiquan Liu* and Lu Fang \\ Department of Radiology, Huadong Hospital, Fudan University, Republic of China}

*Corresponding author: Haiquan Liu, Department of Radiology, Huadong Hospital, Fudan University, 221W. Yan'an Road, Shanghai, People’s Republic of China, 200040, Fax: +86-2162480234; Email: liuhaiquan8811@163.com

\section{Research Article}

Volume 5 Issue 1

Received Date: April 26, 2021

Published Date: June 23, 2021

DOI: $10.23880 /$ crij-16000186

\section{Abstract}

Objective to investigate the radiological features of pulmonary mucinous carcinoma on HRCT (High-resolution Computed Tomography).

Materials and methods: The HRCT features of the 60 cases of pulmonary mucinous carcinoma confirmed by pathology were analyzed, while the clinical data of them also were reviewed retrospectively in our hospital during January 2013 and December 2018.

Results: Our cohort included 39 women and 21 men, age ranged from 34 to 85 years old (mean 63.3 years old). The 60 patients were classified into 2 patterns at HRCT: node/mass $(n=45)$, consolidation $(n=15) .0$ the 60 cases, $34(56.7 \%, 34 / 60)$ patients presented sign of air-like lucency (bubble-like lucency, air bronchogram or cavitation), 27 (45\%, 27/60) patients present the sign of vessel convergence on HRCT. For the 45 cases node/mass lesions, $23(51.1 \%, 23 / 45)$ lesions appeared airlike lucency, 30 cases show lobulations (67\%, 30/45) and 28 (62.2\%, 28/45) cases show speculations. Statistics shows that there were no relationships between sign of air-like lucency and sign of vessel convergence, speculation as well as lobulation were $\mathrm{P}=0.2012, \mathrm{P}=0.2875$ and $\mathrm{P}=0.1337$, respectively.

Conclusion: On HRCT, a pulmonary mucinous carcinoma might appear sign of air-like or vessel convergence, while signs of lobulation and speculation might appear in lesions showed as nodule/mass pattern. Sign of air-like lucency was independent from signs of vessel convergence, lobulation and speculation.

Keywords: Pulmonary Mucinous Carcinoma; HRCT

Abbreviations: HRCT: High-Resolution Computed Tomography; BAC: Bronchioloalveolar Carcinoma; IASLC: Lung Cancer; ATS: American Thoracic Society; ERS: European Respiratory Society; IMA: Invasive Mucinous Adenocarcinoma; CT: Computed Tomography.

\section{Introduction}

Lung cancer continues to be the major cause of cancerrelated mortality worldwide. Non-small lung cancer (NSLC) accounts for $80 \%$ histological type [1]. Invasive mucinous adenocarcinoma was formerly diagnosed as mucinous bronchioloalveolar carcinoma (BAC). In 2011, the International Association for the Study of Lung Cancer (IASLC), American Thoracic Society (ATS) and European Respiratory Society (ERS) proposed a new classification system for lung adenocarcinoma [2]. In this system, invasive mucinous adenocarcinoma (IMA) was recognized as a variant adenocarcinoma subtype. Invasive mucinous adenocarcinoma has a rare incidence, taking approximately $5 \%$ of all lung adenocarcinoma [3]. Computed Tomography (CT) takes a important role in the diagnosis of lung cancer. 


\section{Clinical Radiology \& Imaging Journal}

In this study we reviewed the CT finding of 60 cases invasive mucinous adenocarcinoma being verified pathologically.

\section{Materials and Methods}

\section{Patients}

This retrospective study was approved by the ethic review board at our hospital. From January 2013 to December 2018, the number of patients who were pathologically diagnosed as lung mucinous adenocarcinoma was 80. Of the 80 patients, 10 patients were excluded from the study because their CT scans were not available and 2 patients were excluded for the pathological diagnosis was minimally invasive mucinous adenocarcinoma. Another 2 patients being identified finally as metastasis lesions from other organ also were excluded from the study. Finally, 66 patients who were pathologically diagnosed as primary invasive lung mucinous adenocarcinoma were left. Of the 66 patients, six patients were discarded from the study for they did not meet the goal of the study as their CT scans showed as diffuse pattern in both lungs. At last, 60 patients were included in the present study.

The mean patient age was 63.3 years with range from 34 to 85 years old, and included 21 men and 39 women.

The medical records of the 60 patients were reviewed. Ten of the 60 patients had symptoms as follow: cough and expectoration in 5 cases, cough and fever in 2 cases, shortness of breath in 2 cases and intermittent chest pain in 1 case. The other 50 cases were asymptomatic and the tumors were found by computed tomography (CT) in health examination.

All of the 60 patients underwent radical resection and the specimens were treated and diagnosed in our hospital.

\section{Image Acquisition}

Preoperative chest CT was performed by using the following four scanners: GE Discovery CT750 HD , 64-slice Light Speed VCT (GE Medical Systems); Somatom Definition flash , Somatom Sensation-16 (Siemens Medical Solutions) with the following parameters: $120 \mathrm{kVp} ; 100-200 \mathrm{mAs}$; pitch, 0.75-1.5; and collimation, 1-1.25 mm, respectively. All imaging data were reconstructed by using a medium sharp reconstruction algorithm with a thickness of 1-1.25 mm.19 of the 60 patients were then administered contrast material after non-contrast enhanced CT scan. In the case of contrastenhanced CT, a bolus of $80-100 \mathrm{~mL}$ of intravenous injection contrast medium (350 mg I/mL; Optiray, Mallinckrodt) was administered at a rate of 3-4 mL/s with the use of a power injector via an 18- or 20-gauge cannula in an antecubital vein. The contrast-enhanced CT scan was acquired 60 seconds after the administration of contrast medium.

\section{Image Evaluation}

CT images were evaluated retrospectively in terms of location, lesion size, solidity, internal characteristics, marginal characteristics, and relation to visceral pleura by two radiologists (H.L with 30 years' experience of radiology and F.L with 10 years' experience of radiology).

The cases were classified into 2 groups according to HRCT manifestations [4,5]: nodule/mass pattern and consolidation pattern. In terms of internal characteristics, the lesion was in homogenous and presented air-like attenuation was recorded. The sign of air-like attenuation might be frank cavitation, central air bronchogram or bubble-like areas of low attenuation. The latter is characterized by small foci of low attenuation in (oval, round, or linear) with the lesion that have been variably reported to represent small foci of cavitation, necrosis, bronchiectasis, or focal emphysema [6]. We called all these signs as air-like lucency. Also, the presence signs of vessel convergence were recorded. Vessel convergence referred to vessels around the tumor gathered together and broke at the tumor or penetrated the tumor [7]. Regarding the marginal characteristics of lesions in nodule/mass pattern, the presence of lobulation or notching of the contour was recorded. We also documented the nodule/mass presence of speculation, which defined by the presence of $2 \mathrm{~mm}$ or thicker linear strands extending from the lesion margin towards lung parenchyma without contacting the pleural surface. Moreover, we also evaluated the CT attenuation values of the lesions on plain CT. The CT attenuation were documented for pixels in a circular region interest ( $5 \mathrm{~mm}$ diameter), which covered the lesions away from the air density area. The mean attenuation values were calculated from 3 randomly selected lesion locations. For the 15 cases also undergoing enhanced CT examination, the regions of interest were kept in the same locations and shapes as in plain CT when evaluating enhanced CT attenuation values. For lesions in nodule/mass pattern, the longest tumor diameters were measured.

\section{Statistical Analyses}

Statistical analysis was performed using SAS 9.4 (SAS Institute Inc., Cary, NC). Differences were evaluated by $\chi 2$ test. Differences with $\mathrm{P}<0.05$ were considered statistically significant.

\section{Results}

Of the 60 lung invasive mucinous adenocarcinoma: 45 cases were classified in nodule/mass pattern (Figures 1-3) and 15 cases were fallen into consolidation pattern 
(Figures $4 \& 5$ ). Twenty-seven of the 60 patients appeared sign of air-like, accounting for $45 \%(27 / 60)$; thirty-four of the 60 appeared sign of vessel convergences, accounting for
$56.7 \%(34 / 60)$. Statistical show that there was no significant relationship between sign of vessel convergence and sign of air-like lucency, $\mathrm{P}=0.2012$ (Table 1).

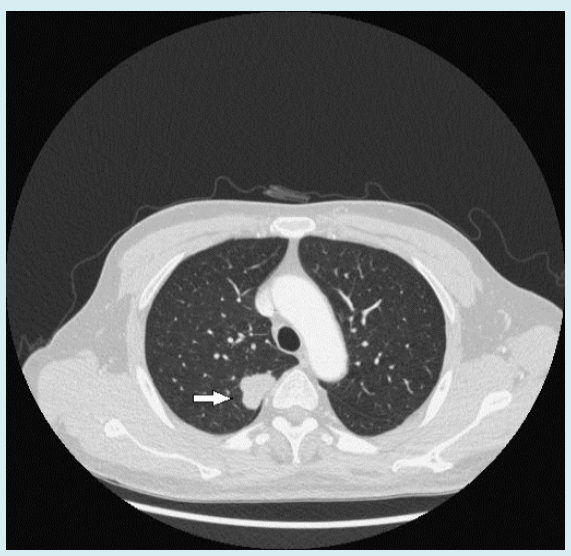

Figure 1: Male, 58 years old. He had no symptom and was found lesion in health examination. CT showed mass in upper lobe of the right lung (arrow.). The mass had lobulation.

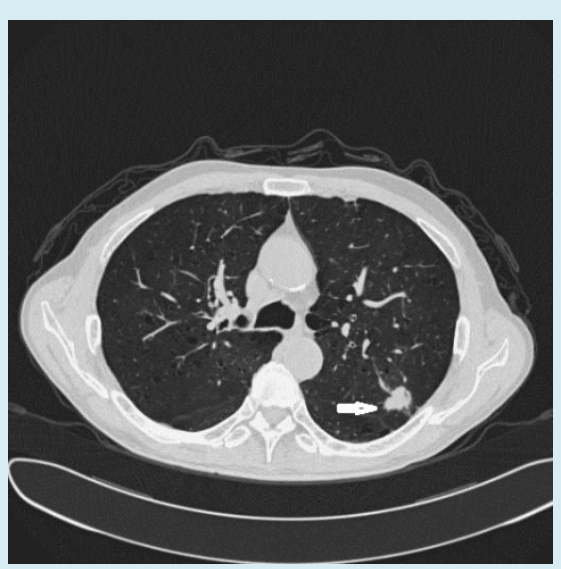

Figure 2: Male, 73 years old. The lesion was found in health examination. CT showed mass in upper lobe of the left lung. The mass had signs of vessel convergence, lobulations and speculations.

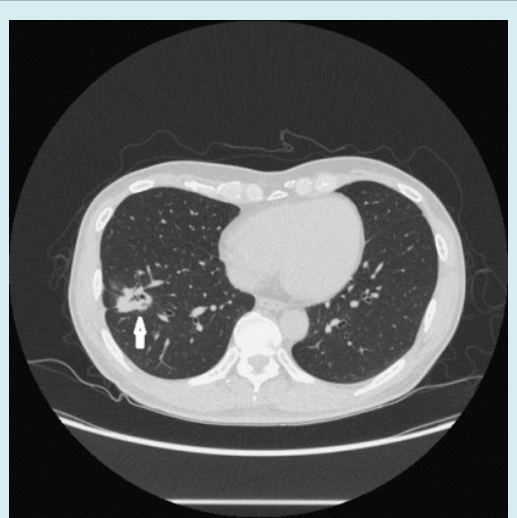

Figure 3: Male, 50 years old. He had cough for 6 weeks. CT showed mass with signs of vessel convergence as well as lobulations and speculations in low lobe of right lung, air-like shadow showing in the mass. 


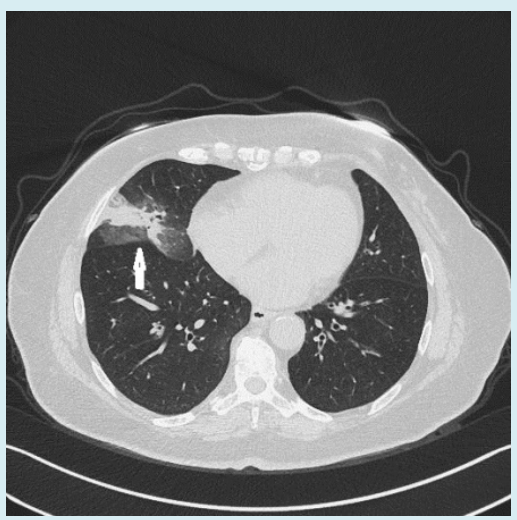

Figure 4: Female, 66 years old. Cough and fever for 1 year. CT showed consolidation with ground glass shadow around in right middle lobe. She was received pulmonary lobectomy and was diagnosed pathologically as pulmonary mucinous carcinoma.

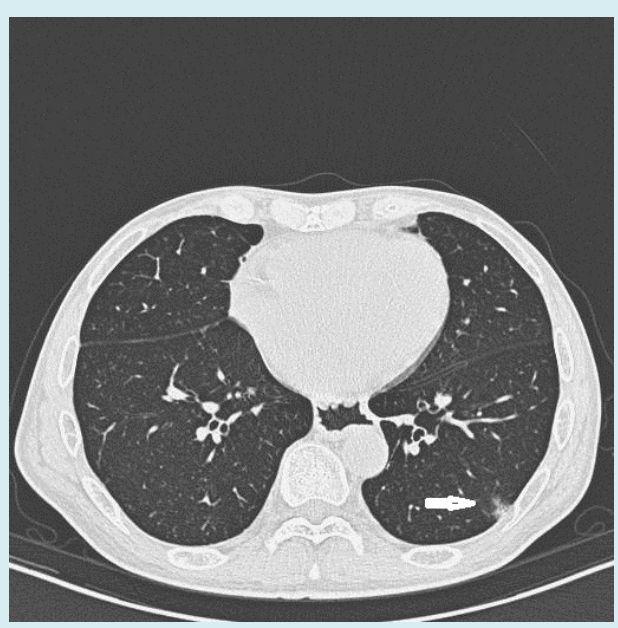

Figure 5: Male, 75 years old. He had no symptom and the lesion ws found in health examination. CT showed patchy consolidation with air-like shadow in low lobe of the left lung.

\begin{tabular}{|c|c|c|c|}
\hline sign & with & without & \\
\hline air-like lucency & $34(56.7 \%)$ & $26(43.3 \%)$ & \\
\hline vessel convergence & $27(45 \%)$ & $33(55 \%)$ & $\mathrm{P}=0.2012$ \\
\hline
\end{tabular}

Table 1: CT sings of air-like lucency and vessel convergence in all cases.

Of the 45 cases showed as in nodule/mass pattern, 23 appeared sign of air-like lucency, accounting for $51 \%$ $(23 / 45)$. In the 45 lesions, the number of lesion showed as lobulation and speculation was 30 and 22, accounting for
$67 \%(30 / 45)$ and $62.2 .6 \%(28 / 45)$, respectively. There was no significant relation between signs of lobulation as well as speculation and sign of air-like lucency, $\mathrm{P}=0.1337$ and $\mathrm{P}=0.2875$, respectively (Table 2).

\begin{tabular}{|c|c|c|c|}
\hline Sign & With & Without & \\
\hline air-like lucency & $23(51.1 \%)$ & $22(48.9 \%)$ & \\
\hline lobulation & $30(67 \%)$ & $15(33 \%)$ & $\mathrm{P}=0.1337$ \\
\hline speculation & $28(62.2 \%)$ & $17(37.8 \%)$ & $\mathrm{P}=0.2875$ \\
\hline
\end{tabular}

Table 2: CT sings of air-like lucency and lobulation as well as speculation in node/mass cases. 


\section{Clinical Radiology \& Imaging Journal}

For the cases of nodule/mass pattern, the lesion diameters ranged from 8 to $72 \mathrm{~mm}$. For the 15 cases of lesions in consolidation pattern, 4 showed ground glass around the lesions (Figure 3).

Of the 60 cases, the average of CT attenuation on plain CT was $13.5 \mathrm{HU}$, ranging from -123 69HU. Fifteen of the 60 cases underwent enhanced CT scans, after enhancement, the increase altitude of CT attenuation was 15-76 Hu, averaging $41.5 \mathrm{HU}$.

The primary radiologist reports of the CT findings were reviewed for all of the 60 patients. According to these reports, 50 cases were diagnosed as lung cancers and 6 cases were diagnosed as inflammatory lesion, while the 5 cases were diagnosed as coexistence of inflammatory and cancer lesion.

\section{Discussion}

Due to the remarkable advance in lung cancer genetics, therapy and radiology in the past decade, the 2004 WHO classification of lung tumors had not met the practical needs. In 2015, WHO published new classification of lung tumors [8]. Compared with 2004 WHO classification, there were multiple major changes for the common lung cancers most of which followed the 2011 lung adenocarcinoma sponsored by the international Association for the study of Lung Cancer (IASLC), American Thoracic Society (ATS), and European Respiratory) Society (ERS), which has essentially been adopted with only minor changes.

In the new classification system, invasive mucinous was taken as a variant of lung adenocarcinoma, with tumor cells showing goblet cell and columnar morphology, abundant apical mucin and basally oriented nuclei [9].

As the mucin has lower density than soft tissue and tumor cells, the mucinous adenocarcinoma are expected to show a relatively low attenuation. In our study, the mean CT attenuation of 60 cases of invasive adenocarcinoma was $13.5 \mathrm{HU}$ and ranged from $-123 \mathrm{HU}$ to $69 \mathrm{HU}$. Miyata, et al. [10] measured the CT values of 22 cases early mucous adenocarcinoma, the means CT value was $25.7 \mathrm{HU}$. They were lower than CT value of normal tissue. Usually, the normal soft shows as attenuation value of approximately $40 \mathrm{HU}$.

Watanabe, et al. [11] observed CT appearances of 40 cases mucinous adenocarcinoma, CT manifestations of the mucinous adenocarcinoma were nodule/ masses or consolidation, ground glass opacity might appear around both types. Miyata, et al. [10] study 22 cases early mucinous adenocarcinoma, they all showed as solid or part-solid nodules. In our study of the 60 cases, 45 cases showed as nodule/mass and 15 cases showed as consolidation, and
4 cases of consolidation type accompanied ground glass around consolidations. The formation of ground glass was probably due to some of the tumor cells growing in lepidic pattern without production of mucin around consolidation. Lepidic growth style of adenocarcinoma showed as ground glass on CT $[12,13]$.

Our study showed that most lung invasive mucinous adenocarcinomas of nodule/mass pattern appeared lobulations and speculations, being similar to other kinds of lung adenocarcinoma [14]. Zwirewich, et al. [6] found that the pathological correlate of lobulation was the presence of nodular excrescence of the tumor at its advancing edge and that the formation of speculation was due to the desmoplastic reaction, resulting in fibrotic strands radiating into the surrounding lung parenchyma. It is interesting that, Miyata, et al. [10] observed pathological factors for high resolution CT in 22 cases of early lung mucinous adenocarcinoma, they found that 21 cases contained central fibrosis.

Studies showed that the sign of air-like lucency was more specific for BAC than benign lesions of lung $[15,16]$. Air-like lucency correlated with (a) frank cavitation, (b) bubblelike areas of low attenuation, (c) patent bronchi producing air bronchogram. Small cavitation pathologically was found to represent focal areas of bronchiectasis in some cases. While bubble-like areas of low attenuation may be due to air-containing bronchi within the mass or can be caused by cystic space within papillary regions of tumor growth in BACs [6]. The open bronchus sign was produced by alveoli filled with tumor and mucus surrounding the aerated bronchi. There were likely narrowing, stretching and expanding in mucinous adenocarcinoma [14]. Zwirewich CV, et al. [6] studied 85 cases solitary nodules $(11$ benign nodules and 75 malignant nodules). They found that sign of bubble-like were more frequently seen in malignant nodules (25\%vs9\%). Sign of bubble-like accounted for $50 \%(7 / 14)$ in BAC. Miyata, et al. [10] study 22 cases early mucinous adenocarcinoma, the bubble-like sign appeared in $86.4 \%$ cases $(19 / 22)$. Our result showed that the sign air-like lucency accounted for $45 \%(27 / 60)$, being similar to above studies.

The air-like lucency was probably caused by other mechanisms. Ohba, S, et al. [17] reported a 47-year-old woman in whom chest radiography showed bilateral, diffuse reticulonodular infiltrates with multiple cysts $3-10 \mathrm{~mm}$ in diameter and numerous small nodule with central pin-holes. At autopsy, bilateral cystic destruction of the pulmonary parenchyma was seen. The pathogenesis of cyst formation was thought to be due to the two major mechanisms: central necrosis with the nodule and, more commonly, check-valve obstruction at the terminal bronchiolar level. Thickened fibrous terminal bronchioles were found in some sections, which could account for the check-valve obstruction and 


\section{Clinical Radiology \& Imaging Journal}

formation of emphysematous cysts. Wethisbrod, et al. [18] reported that the smaller cyst lesions arouse because of tumor growth through post-obstructive bronchiectasis in diffuse bronchioloalveolar carcinoma. In some instances of multiple cysts the neoplasm could have invaded preexisting cysts.

One or several vessel around lesions reach, structure and pass through tumors is called vessel convergence sign. $\mathrm{Hu} \mathrm{H}$, et al. [19] observed 82 malignant and 30 benign glass nodules, Seeman, et al. [20] analyzed 80 malignant nodules and 23 benign nodules, they both found that the vessel convergence more often appeared in malignant lesions and it had value in distinguishing malignant lesions from benign lesions. Meniga IN, et al. [21] analyzed the relationship between survival and sing of vessel convergence; they found that the sign of vessel convergence was the index of poor progenies. At present study,, the cases who appeared vessel convergence was 34 , accounting for $56.7 \%$ (34/60).

In our study, we analyzed the relationship between the sign of air-like lucency and the sign of vessel convergence, we found no statistical significance. Similarly, there was no statistical significance between the sign of air-like lucency and the signs of lobulation as well as speculation for lesion in nodule/mass pattern. These results implied that the sign of air-like lucency was a potential index for diagnosing invasive mucinous adenocarcinoma independent from vessel convergence, lobule and speculation.

\section{References}

1. Bueno R, Hughes E, Wagner S, Gutin AS, Lanchbury JS, et al. (2015) Validation of a molecular and pathological model for five-year mortality risk in patients with early stage lung adenocarcinoma. J Thorac Oncol 10(1): 67-73.

2. Travis WD, Brambilla E, Noguchi M, Nicholson AG, Geisinger KR, et al. (2011) International association for the study of lung cancer/american thoracic society/european respiratory society international multidisciplinary classification of lung adenocarcinoma. J Thorac Oncol 6(2): 244-285.

3. Shim HS, Kenudson M, Zheng Z, Liebers M, Cha YJ, et al. (2015) Unique Genetic and Survival Characteristics of Invasive Mucinous Adenocarcinoma of the Lung. J Thorac Oncol 10(8): 1156-1162.

4. MacMahon H, Naidich DP, Goo JM, Lee KS, Leung ANC, et al. (2017) Guidelines for Management of Incidental Pulmonary Nodules Detected on CT Images: From the Fleischner Society 2017. Radiology 284(1): 228-243.

5. Bueno J, Landeras L, Chung JH (2018) Updated Fleischner
Society Guidelines for Managing Incidental Pulmonary Nodules: Common Questions and Challenging Scenarios. Radiographics 38(5): 1337-1350.

6. Zwirewich CV, Vedal S, Miller RR, Muller NL (1991) Solitary pulmonary nodule: high-resolution CT and radiologic-pathologic correlation. Radiology 179(2): 469-476.

7. Ma J, Yang YL, Wang Y, Zhang XW, Gu XS, et al. (2017) Relationship between computed tomography morphology and prognosis of patients with stage I nonsmall cell lung cancer. Onco Targets Ther 10: 2249-2256.

8. Travis WD, Brambilla E, Nicholson AG, Yatabe Y, Austin JHM, et al. (2015) The 2015 World Health Organization Classification of Lung Tumors: Impact of Genetic, Clinical and Radiologic Advances Since the 2004 Classification. J Thorac Oncol 10(9): 1243-1260.

9. Boland JM, Maleszewski JJ, Wampfler JA, Voss JS, Kipp BR, et al. (2018) Pulmonary invasive mucinous adenocarcinoma and mixed invasive mucinous/ nonmucinous adenocarcinoma-a clinicopathological and molecular genetic study with survival analysis. Hum Pathol 71: 8-19.

10. Miyata N, Endo M, Nakajima T, Kojima H, Maniwa T, et al. (2015) High-resolution computed tomography findings of early mucinous adenocarcinomas and their pathologic characteristics in 22 surgically resected cases. Eur J Radiol 84(5): 993-997.

11. Watanabe H, Saito H, Yokose T, Sakuma Y, Murakami S, et al. (2015) Relation between thin-section computed tomography and clinical findings of mucinous adenocarcinoma. Ann Thorac Surg 99(3): 975-981.

12. Austin JH, Garg K, Aberle D, Yankelevitz D, Kuriyama $\mathrm{K}$, et al. (2013) Radiologic implications of the 2011 classification of adenocarcinoma of the lung. Radiology 266(1): 62-71.

13. Park CM, Goo JM, Lee HJ, Lee CH, Chun EJ, et al. (2007) Nodular ground-glass opacity at thin-section CT: histologic correlation and evaluation of change at followup. Radiographics 27(2): 391-408.

14. Furuya K, Murayama S, Soeda H, Murakami J, Ichinose $Y$, et al. (1999) New classification of small pulmonary nodules by margin characteristics on high-resolution CT. Acta Radiol 40(5): 496-504.

15. Gaeta M, Caruso R, Blandino A, Bartiromo G, Scribano E, et al. (1999) Radiolucencies and cavitation in bronchioloalveolar carcinoma: CT-pathologic 
correlation. Eur Radiol 9(1): 55-59.

16. Kuhlman JE, Fishman EK, Kuhajda FP, Meziane MM, Khouri NF, et al. (1988) Solitary bronchioloalveolar carcinoma: CT criteria. Radiology 167(2): 379-382.

17. Ohba S, Takashima T, Hamada S, Kitagawa M (1972) Multiple cystic cavitary alveolar-cell carcinoma. Radiology 104(1): 65-66.

18. Weisbrod GL, Towers MJ, Chamberlain DW, Herman SJ, Matzinger FR (1992) Thin-walled cystic lesions in bronchioalveolar carcinoma. Radiology 185(2): 401405.

19. Hu H, Wang Q Tang H, Xiong L, Lin Q (2016) Multi-

\section{Clinical Radiology \& Imaging Journal}

slice computed tomography characteristics of solitary pulmonary ground-glass nodules: Differences between malignant and benign. Thorac Cancer 7(1): 80-87.

20. Seemann MD, Staebler A, Beinert T, Dienemann H, Obst B, et al. (1999) Usefulness of morphological characteristics for the differentiation of benign from malignant solitary pulmonary lesions using HRCT. Eur Radiol 9(3): 409417.

21. Meniga IN, Tiljak MK, Ivankovic D, Aleric I, Zekan M, et al. (2010) Prognostic value of computed tomography morphologic characteristics in stage I non-small-cell lung cancer. Clin Lung Cancer 11(2): 98-104. 